\title{
Integration of finite displacement interface element in reference and current configurations
}

\author{
Francesco Parrinello • Guido Borino
}

Received: date / Revised version: date

\begin{abstract}
In the present paper the non-linear behaviour a solid body with embedded cohesive interfaces is examined in a finite displacements context. The principal target is the formulation of a two dimensional interface finite element which is referred to a local reference frame, defined by normal and tangential unit vectors to the interface middle surface. All the geometric operators, such as the interface elongation and the reference frame, are computed as function of the actual nodal displacements. The constitutive cohesive law is defined in terms of Helmholtz free energy for unit of undeformed interface surface and, in order to obtain the same nodal force vector and stiffness matrix by the two integration schemes, the cohesive law in the deformed configuration is defined in terms of Cauchy traction, as a function of separation displacement and of interface elongation. Explicit expression of the nodal force vector is integrated either over the reference configuration or over the current configuration, which is shown to produce the same analytical finite element operators. No differences between the integration carried out in the reference and in the current configuration are shown, provided that elongation of the interface is taken in to account.
\end{abstract}

Keywords finite displacement - cohesive interface - integration - reference configuration-current configuration

\section{Introduction}

Cohesive Zone Models (CZM) are nowadays one of the most powerful theoretical and computational tools able to describe non-linear fracture processes in solid mechanics. Structural failure for quasi-brittle materials is often characterized by the formation, development and coalescence of micro-cracks, which

Dipartimento di Ingegneria Civile, Ambientale, Aerospaziale, dei Materiali. Università di Palermo, Viale delle Scienze, Ed. 8, 90128 Palermo, Italy E-mail: francesco.parrinello@unipa.it 
produce a strain localization with a subsequent formation of a separation surface where cohesive forces tend to progressively vanish up to creation of a discontinuity or fracture surface. For classical fracture mechanics problems the actual geometric position, as well as the direction of propagation of the cohesive surface, are themselves part of the non-linear problem; however, there is a large number of situations in which the location of the potential decohesion surface can be established a priori. For this kind of problems it is sufficient to introduce an interface surface embedded inside the continuum solid with the intent to drive the potential fracture development.

In small strain theory interface constitutive relations usually employ cohesive fracture approach by means of damage mechanics theory. The fields of application are extremely wide, e.g.: interlaminar fracture for composite materials [1], failure modes of masonry structures [2,3], breaking mechanisms for adhesive or welded joints [4], fragmentation processes [5,6] and many others.

Even if the most common interface formulations are developed under the hypothesis of small strains and small displacements, their application to geometrically non-linear problems is of great interest. Few contributions on cohesive interface in large displacement are available in literature. Recently, in [7-9] some possible limits and deficiencies have been discussed for large displacement interfaces. In [7] the state of the art of cohesive models for the material separation such as cracks and delamination is presented, focusing on thermodynamics and variational consistency, and showing that many proposed models do not satisfy fundamental requirements, such as thermodynamic principles, frame invariance or equilibrium conditions. Such problems are especially encountered for anisotropic models in geometrically non-linear context.

One of the first contribution on the subject is presented in [1] where delamination in composite structures is analysed in a finite displacement setting. A pioneering interface finite element formulation for large displacement three-dimensional problems is developed in [10], where the first Piola-Kirchoff traction vector is defined as a function of the normal and tangential separation displacement components, with respect to the middle surface in the current configuration. A further quite effective approach is the corotational interface formulation proposed in [11] for modelling buckling and delamination phenomena in composite materials. The corotational approach properly predicts the non-linear geometric effects due to the rotation of the interface, but the effect due to the elongation of the interface is not taken into account.

In [12] the constitutive modelling of fibrillation phenomenon, common of soft polymer coating is proposed. Two different formulation are developed, respectively for the small deformation condition and for the large deformation one, with a smooth transition from the former to the latter.

In [9] the lack of rotational equilibrium in the deformed configuration is analysed for some existing CZMs, showing that sufficient condition for rotational equilibrium to be satisfied is that traction vector and separation displacement vector are co-axial, such as for isotropic interface models. Such a condition is often not properly fulfilled by CZMs devoted to produce two dif- 
ferent fracture energies in mode I and in mode II fracture conditions, in finite strains and finite displacements regime [13-15].

In [8] CZMs are analysed in large displacements and large strains conditions. The interface models are also evaluated with respect to thermodynamic consistency, balance of angular momentum and frame invariance. It is shown that in elastic regime only isotropic models, with traction vector co-axial to separation displacement vector, fulfill all physical principles. The same condition is analysed for an elasto-plastic CZM developing an isotropic constitutive model, which is defined as a set of elasto-plastic truss elements connecting the two edges of the interface. Moreover, the authors proposed a second formulation, assuming that the ends of the elasto-plastic truss elements can also plastically slide on the crack surface. The traction vector results to be aligned to the connected points, but not aligned to the separation displacement vector. This approach represents a relaxed condition for the satisfaction of the balance of angular momentum, with respect to the original formulation of traction vector aligned to the separation displacement vector, proposed in [9].

The paper [16] proposes an interface element formulation for geometrical non-linearity and material non-linearity, developed in the reference configuration. The constitutive model is defined on the local reference frame, defined by normal axis and tangential axis with respect to the middle surface in the current configuration.

In [17] a four nodes interface element has been analysed and tested under large displacement conditions. The interface constitutive model used for the numerical simulations is the potential based model proposed in [18] and the finite element operators (stiffness matrix and nodal force vector) have been evaluated numerically by both the Gauss quadrature rule and by the Newton-Cotes quadrature rule. Moreover, both the integration over the reference surface and the integration over the deformed middle surface have been considered for the same constitutive model, showing significant difference between the two integration schemes. The latter surprising result is due to some kind of hidden numerical approximation when integration is performed over the current configuration, as supposed in [17] .

In the present paper the interface formulation is rigorously developed under finite displacement conditions, assuming as local reference frame for the constitutive model, normal and tangential axes to the middle surface.

The geometric operators in the current configuration, such as the normal and tangential axes to the middle surface and elongation of the middle surface, are defined as functions of nodal displacements. The formulation here proposed regards $2 \mathrm{D}$ problems. The extension to $3 \mathrm{D}$ problems is, of course, possible but it is not completely straightforward. The main difficulties for the extension stand in the geometrical treatment of a moving interface surface embedded in a $3 \mathrm{D}$ continuum space.

Nodal force vector and consistent stiffness matrix are computed for a two-dimensional interface element in either reference and current integration schemes and the relation between the cohesive laws in the reference configuration and in the current configuration is defined. It is shown that the cohesive 
law can be defined in the current configuration in terms of Cauchy traction vector, which is defined as function of normal and tangential components of separation displacement, and as function of the interface elongation.

The proposed interface formulation has been implemented in the open source finite element code FEAP [19] with reference to the cohesive-frictional interface model proposed in [20,21]. The results of the numerical simulations for an end-notched double cantilever beam test are proposed and compared with analytical solutions.

\section{Finite displacement mechanical problem with interface}

\subsection{Interface kinematics}

Let a solid body be considered occupying the region $\Omega_{0} \subset \mathbb{R}^{3}$ in the reference configuration and let the body be characterized by an embedded surface $\Gamma_{0}$ in which discontinuity of the deformed configuration field may develop. The surface $\Gamma_{0}$ splits the solid in two parts $\Omega_{0}^{+}$and $\Omega_{0}^{-}$with shared boundary $\Gamma_{0}$. The normal vector $\boldsymbol{n}_{0}$ to the surface $\Gamma_{0}$ is positive if oriented from $\Omega_{0}^{-}$to $\Omega_{0}^{+}$. Each material point $\boldsymbol{X} \in \Omega_{0}$ undergoes deformation following the nonlinear mapping $\boldsymbol{x}=\boldsymbol{x}(\boldsymbol{X}, t)$, which is smooth and continuous with one-to-one relation for all material points in $\Omega_{0}$, with the exception of the material points $\boldsymbol{X} \in \Gamma_{0}$ where a multiple mapping can exist (see Figure 1). The deformed configuration of a point lying on the surface $\Gamma_{0}$ in the reference configuration can be defined by using the following notation

$$
\begin{aligned}
& \boldsymbol{x}^{+}=\boldsymbol{x}^{+}(\boldsymbol{X}, t), \text { with } \boldsymbol{X} \in \Gamma_{0} \subset \partial \Omega_{0}^{+}, \\
& \boldsymbol{x}^{-}=\boldsymbol{x}^{-}(\boldsymbol{X}, t), \text { with } \boldsymbol{X} \in \Gamma_{0} \subset \partial \Omega_{0}^{-},
\end{aligned}
$$

which is represented in Fig. 1

In the current configuration the two edges of the embedded surface are separate in two distinct surfaces $\Gamma^{+}$and $\Gamma^{-}$, with $\boldsymbol{x}^{+} \in \Gamma^{+}$and $\boldsymbol{x}^{-} \in \Gamma^{-}$. Moreover, the middle surface $\Gamma_{m}$, after $[10,16]$, is defined in the current configuration as the set of points $\boldsymbol{x}_{m}$, which are defined by the following equation

$$
\boldsymbol{x}_{m}(\boldsymbol{X}, t):=\frac{1}{2}\left[\boldsymbol{x}^{+}(\boldsymbol{X}, t)+\boldsymbol{x}^{-}(\boldsymbol{X}, t)\right] \text { with } \boldsymbol{X} \in \Gamma_{0}
$$

and the axes $\boldsymbol{n}$ and $\boldsymbol{t}$, which are respectively normal and tangential to the middle surface $\Gamma_{m}$ at point $\boldsymbol{x}_{m}$, are assumed as local reference frame of the interface cohesive law. In the initial configuration the middle surface coincides with the embedded surface $\Gamma_{0}$.

The displacement field, defined as

$$
\boldsymbol{u}(\boldsymbol{X}, t)=\boldsymbol{x}(\boldsymbol{X}, t)-\boldsymbol{X}
$$




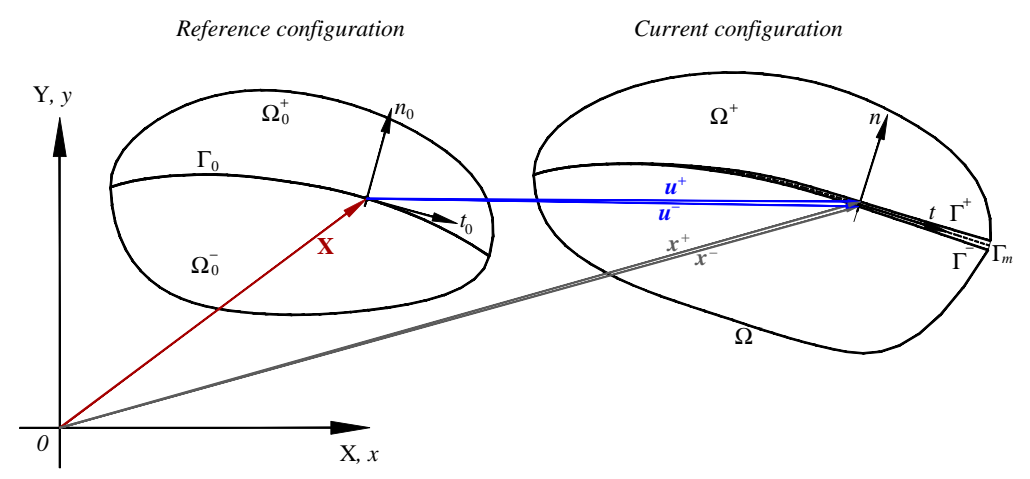

Fig. 1 Representation of the one-to-one mapping between current and reference configurations

can be discontinuous on the surface $\Gamma_{0}$, with two different values on the domain $\Omega_{0}^{+}$and on the domain $\Omega_{0}^{-}$, that is

$$
\begin{aligned}
& \boldsymbol{u}^{+}=\boldsymbol{u}^{+}(\boldsymbol{X}, t), \text { with } \boldsymbol{X} \in \Gamma_{0} \subset \partial \Omega_{0}^{+}, \\
& \boldsymbol{u}^{-}=\boldsymbol{u}^{-}(\boldsymbol{X}, t), \text { with } \boldsymbol{X} \in \Gamma_{0} \subset \partial \Omega_{0}^{-} .
\end{aligned}
$$

The separation displacement at points $\boldsymbol{X} \in \Gamma_{0}$ between the two edges of the embedded surface is

$$
\llbracket \boldsymbol{u}(\boldsymbol{X}, t) \rrbracket=\boldsymbol{x}^{+}(\boldsymbol{X}, t)-\boldsymbol{x}^{-}(\boldsymbol{X}, t)=\boldsymbol{u}^{+}(\boldsymbol{X}, t)-\boldsymbol{u}^{-}(\boldsymbol{X}, t) .
$$

The deformation gradient tensor $\boldsymbol{F}$ and the relevant jacobian determinant are defined as

$$
\begin{aligned}
\boldsymbol{F}(\boldsymbol{X}, t) & =\frac{\partial \boldsymbol{x}(\boldsymbol{X}, t)}{\partial \boldsymbol{X}}, \\
J(\boldsymbol{X}, t) & =\operatorname{det}(\boldsymbol{F}(\boldsymbol{X}, \mathrm{t})) .
\end{aligned}
$$

The deformation gradient $\boldsymbol{F}$, following the condition (1) is not continuous on the embedded surface $\Gamma_{0}$, moreover it does not affects the cohesive behaviour of the connecting interface between the two bodies.

\subsection{Balance laws}

The internal surface $\Gamma_{0}$ is locus of cohesive tractions, which can be defined by a specific cohesive interface constitutive law as a non-linear function of the separation displacement $\mathbf{T}:=\mathbf{T}(\llbracket \boldsymbol{u} \rrbracket)$.

In $[8,9]$ the linear and angular balance momentum of the interface, with a finite thickness in the current configuration, has been deeply analysed and, as generally accepted in classical cohesive zone modelling, the membrane-like 
traction on the boundary surface $\Gamma_{b}$ is neglected (see Figure 2). Under such a condition, the balance of linear momentum can be written in the reference configuration in the following form

$$
T^{+}=-T^{-}=T
$$

where $\boldsymbol{T}^{+}$and $\boldsymbol{T}^{-}$are the First Piola-Kirchhoff (FPK) traction vectors and are related to the Cauchy traction vectors $\boldsymbol{S}^{+}$and $\boldsymbol{S}^{-}$through the relations

$$
\boldsymbol{T}^{+} d \Gamma_{0}=\boldsymbol{S}^{+} d \Gamma^{+} \text {and } \boldsymbol{T}^{-} d \Gamma_{0}=\boldsymbol{S}^{-} d \Gamma^{-} .
$$

By neglecting the membrane-like tractions on the boundary surfaces $\Gamma_{b}$, the balance of angular momentum can be written in the reference configuration by the following vector cross product:

$$
\llbracket u \rrbracket \times T=\mathbf{0},
$$

which means that sufficient condition for the enforcement of balance of angular momentum is that traction vector $\boldsymbol{T}$ is aligned to the separation displacement $\llbracket \boldsymbol{u} \rrbracket$. Balance of angular momentum requires isotropic cohesive relations (coaxiality between displacement jump and traction vector) or, alternatively, one of the two member in Eq. (9) remains small or vanishes. The displacement jump $\llbracket \boldsymbol{u} \rrbracket$ for relevant cohesive state can be assumed small enough to assure Eq. (9) to hold, whereas traction $\boldsymbol{T}$ become negligible for large displacement jump up to complete delamination condition. The latter condition naturally applies for many material cohesive interface. Namely, the balance of angular momentum can be satisfied by assuming an isotropic elastic behaviour or by assuming the hypothesis of small separation displacement before full debonding.

Reference configuration

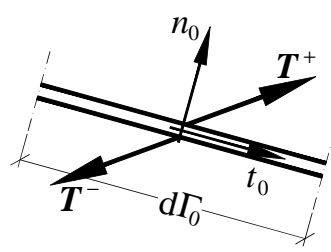

\section{Current configuration}

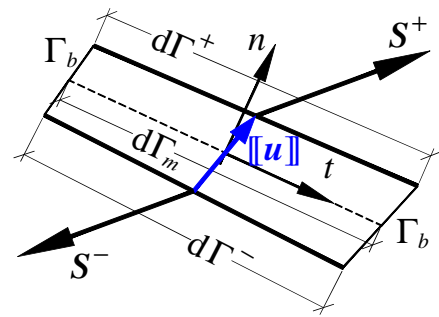

Fig. 2 Representative Surface Element in the current configuration and in the reference one. Piola-kirchoff traction $\boldsymbol{T}$ and Cauchy traction $\boldsymbol{S}$ are represented respectively in the reference configuration and in the current one.

The constitutive behaviour of the interface surface $\Gamma_{0}$ in the elastic regime, and in particular in the post-elastic one, is strongly affected by the delamination mode, with different responses in opening and sliding modes. As a 
consequence, the cohesive consitutive law has to be defined in a local reference frame with normal and tangential axes, with respect to embedded surface $\Gamma$ in the current configuration, which is not unique, due to the discontinuity of displacement field. The solution generally assumed in literature, and initially proposed in [10], is to consider as unique deformed configuration of the surface $\Gamma_{0}$ the middle surface $\Gamma_{m}$ defined in Eq. (2) and represented in Fig. 1 and in Fig. 2 with the relevant local frame, defined by the tangential and normal axes $(\boldsymbol{t}, \boldsymbol{n})$. The difference between middle surface $\Gamma_{m}$ and actual deformed surfaces $\Gamma^{+}$and $\Gamma^{-}$is generally very small for not fully debonded interfaces. In fact, excluding specific cases such as fibrillation phenomenon, the separation displacement vector before full debonding is small compared to size of the reference surface $\Gamma_{0}$. When the interface is fully debonded the spatial position of middle surface $\Gamma_{m}$ can be significantly different from the two deformed surfaces $\Gamma^{+}$and $\Gamma^{-}$, but without any traction transmitted between them.

\subsection{Principle of Virtual Work}

The Principle of Virtual Work (PVW) for a domain with an internal embedded surface $\Gamma_{0}$ can be written in the reference configuration in the following form

$$
\int_{\Omega_{0}} \boldsymbol{P}: \delta \boldsymbol{F} d \Omega_{0}+\int_{\Gamma_{0}} \boldsymbol{T} \cdot \llbracket \delta \boldsymbol{u} \rrbracket d \Gamma_{0}=P_{e x t}
$$

where $\boldsymbol{P}=J \boldsymbol{\sigma} \boldsymbol{F}^{-T}$ is the first Piola-Kirchhoff stress tensor, with $\boldsymbol{\sigma}$ the Cauchy stress tensor, $\delta \boldsymbol{F}$ is a virtual deformation gradient, $\llbracket \delta \boldsymbol{u} \rrbracket$ is a virtual separation displacement and $P_{\text {ext }}$ is the external virtual work.

The PVW can also be written in the current configuration as

$$
\int_{\Omega} \boldsymbol{\sigma}: \delta \boldsymbol{\epsilon} d \Omega+\int_{\Gamma^{+}} \boldsymbol{S}^{+} \cdot \delta \boldsymbol{u}^{+} d \Gamma^{+}+\int_{\Gamma^{-}} \boldsymbol{S}^{-} \cdot \delta \boldsymbol{u}^{-} d \Gamma^{-}=P_{e x t}
$$

where $\delta \boldsymbol{\epsilon}=\frac{1}{2}\left(\delta \boldsymbol{F} \cdot \boldsymbol{F}^{-1}+\boldsymbol{F}^{-T} \cdot \delta \boldsymbol{F}^{T}\right)$ is the virtual variation of the deformation tensor. Under the hypothesis of small separation displacement before full debonding, the two surfaces $\left(\Gamma^{+}\right.$and $\left.\Gamma^{-}\right)$, in the deformed configuration, can be assumed to be sufficiently close to the middle surface $\Gamma_{m}$. On the contrary, at fully debonded points of the interface, the two surfaces are different one of each other, but with null tractions $\mathbf{S}^{+}$and $\mathbf{S}^{-}$acting on such surfaces. Therefore, the PVW in the current configuration in Eq. (11) can be rewritten in the following form

$$
\int_{\Omega} \boldsymbol{\sigma}: \delta \boldsymbol{\epsilon} d \Omega+\int_{\Gamma_{m}} \boldsymbol{S} \cdot \llbracket \delta \boldsymbol{u} \rrbracket d \Gamma_{m}=P_{e x t}
$$

where interface tractions in the current configuration are $\boldsymbol{S}=\boldsymbol{S}^{+}=-\boldsymbol{S}^{-}$, with $d \Gamma_{m} \cong d \Gamma^{+} \cong d \Gamma^{-}$. The relation between reference and current traction vectors in Eq.(8a, b) can be simplified in the following form

$$
\boldsymbol{T} d \Gamma_{0}=\boldsymbol{S} d \Gamma_{m} .
$$


In the following the hypothesis of small separation displacement before full debonding is adopted.

The cohesive law is defined in terms of normal and tangential components $\left(T_{n}=\boldsymbol{T} \cdot \boldsymbol{n}\right.$ and $\left.T_{t}=\boldsymbol{T} \cdot \boldsymbol{t}\right)$ of the FPK traction vector, with respect to the middle surface $\Gamma_{m}$, and they are assumed as functions of the normal and tangential components of the separation displacement $u_{n}=\llbracket \boldsymbol{u} \rrbracket \cdot \boldsymbol{n}$ and $u_{t}=$ $\llbracket \boldsymbol{u} \rrbracket \cdot t$. The FPK traction vector, the Cauchy traction vector and the separation displacement vector are defined in the local reference frame $(\boldsymbol{t}, \boldsymbol{n})$ by the following relations

$$
\begin{gathered}
\tilde{\boldsymbol{T}}=\left[\begin{array}{l}
T_{t} \\
T_{n}
\end{array}\right]=\mathbf{G} \cdot \boldsymbol{T} \\
\tilde{\boldsymbol{S}}=\left[\begin{array}{l}
S_{t} \\
S_{n}
\end{array}\right]=\mathbf{G} \cdot \boldsymbol{S} \\
\llbracket \tilde{\boldsymbol{u}} \rrbracket=\left[\begin{array}{l}
u_{t} \\
u_{n}
\end{array}\right]=\mathbf{G} \cdot \llbracket \boldsymbol{u} \rrbracket
\end{gathered}
$$

where $\mathbf{G}=[\boldsymbol{t}, \boldsymbol{n}]^{T}$ is the rotation matrix which relates the local coordinate system to the global one. Because of the large displacement regime the rotation matrix $\mathbf{G}$ has to be considered as function of the displacement field $\boldsymbol{u}$. The cohesive law is defined in the local frame as a function $\tilde{\boldsymbol{T}}:=\tilde{\boldsymbol{T}}(\llbracket \tilde{\boldsymbol{u}} \rrbracket)$ and it can easily proved that the cohesive law satisfies the principle of material frame indifference (see [10]).

The virtual work done by cohesive traction for a virtual body displacement $\delta \boldsymbol{u}$ in Eq.(10) can be computed both in the local reference frame $(\boldsymbol{t}, \boldsymbol{n})$ and in the global one $(\mathrm{X}, \mathrm{Y})$. A virtual body displacement field $\delta \boldsymbol{u}$ produces a virtual separation displacement $\llbracket \delta \boldsymbol{u} \rrbracket=\delta \boldsymbol{u}^{+}-\delta \boldsymbol{u}^{-}$in the global reference frame $(\mathrm{X}, \mathrm{Y})$, whereas the virtual separation displacement produced in the local reference frame $(\boldsymbol{t}, \boldsymbol{n})$ can be obtained by applying differentiation chain rule to $\mathrm{Eq} \cdot(14 \mathrm{c})$, that is

$$
\llbracket \delta \tilde{\boldsymbol{u}} \rrbracket=\frac{\partial \llbracket \tilde{\boldsymbol{u}} \rrbracket}{\partial \boldsymbol{u}} \cdot \delta \boldsymbol{u}=\left(\frac{\partial \mathbf{G}}{\partial \boldsymbol{u}} \cdot \delta \boldsymbol{u}\right) \cdot \llbracket \boldsymbol{u} \rrbracket+\mathbf{G} \cdot \llbracket \delta \boldsymbol{u} \rrbracket .
$$

So the virtual work done by cohesive traction for a virtual displacement $\delta \boldsymbol{u}$ in the local frame $(\boldsymbol{t}, \boldsymbol{n})$ and in the global one (X, Y) can be written as

$$
\boldsymbol{T} \cdot \llbracket \delta \boldsymbol{u} \rrbracket=\tilde{\boldsymbol{T}} \cdot \llbracket \tilde{\delta} \boldsymbol{u} \rrbracket=\tilde{\boldsymbol{T}} \cdot\left[\left(\frac{\partial \mathbf{G}}{\partial \boldsymbol{u}} \cdot \delta \boldsymbol{u}\right) \cdot \llbracket \boldsymbol{u} \rrbracket+\mathbf{G} \cdot \llbracket \delta \boldsymbol{u} \rrbracket\right]
$$

and the substitution of Eq. (16) in Eq. (10) allows to rewrite the PVW in the reference configuration in the following form

$$
\int_{\Omega_{0}} \boldsymbol{P}: \delta \boldsymbol{F} d \Omega_{0}+\int_{\Gamma_{0}} \tilde{\boldsymbol{T}} \cdot\left[\left(\frac{\partial \mathbf{G}}{\partial \boldsymbol{u}} \cdot \delta \boldsymbol{u}\right) \cdot \llbracket \boldsymbol{u} \rrbracket+\mathbf{G} \cdot \llbracket \delta \boldsymbol{u} \rrbracket\right] d \Gamma_{0}=P_{e x t},
$$

and the PVW can be written in the current configuration as

$$
\int_{\Omega} \boldsymbol{\sigma}: \delta \boldsymbol{\epsilon} d \Omega+\int_{\Gamma_{m}} \tilde{\boldsymbol{S}} \cdot\left[\left(\frac{\partial \mathbf{G}}{\partial \boldsymbol{u}} \cdot \delta \boldsymbol{u}\right) \cdot \llbracket \boldsymbol{u} \rrbracket+\mathbf{G} \cdot \llbracket \delta \boldsymbol{u} \rrbracket\right] d \Gamma_{m}=P_{e x t} .
$$




\section{Interface constitutive model}

Interface constitutive relations has to be derived on the basis of thermodynamic restrictions, which require the definition of an Helmholtz free energy density (per unit of undeformed surface $\Gamma_{0}$ ) and ensuring the non negativity of the mechanical dissipation density for any possible loading path. In the current configuration, due to the variation of the deformed surface area with respect to the undeformed one, the cohesive law has to be defined as function of separation displacement and current surface area $d \Gamma_{m}$, that is $\tilde{\boldsymbol{S}}:=\tilde{\boldsymbol{S}}\left(\llbracket \tilde{\boldsymbol{u}} \rrbracket, d \Gamma_{m}\right)$.

The elastic-damage constitutive model is based on the same cohesive formulation proposed in [20], with bi-linear traction separation law. The Helmholtz free energy density function for unit of undeformed surface $\Gamma_{0}$ is introduced as

$$
\psi\left(u_{t}, u_{n}, \omega, \eta\right)=\frac{1}{2}(1-\omega) \llbracket \tilde{\boldsymbol{u}} \rrbracket \cdot \mathbf{k}_{e l}^{0} \cdot \llbracket \tilde{\boldsymbol{u}} \rrbracket+\psi_{i n}(\eta),
$$

where $\omega$ (with $0 \leq \omega \leq 1$ ) is the damage parameter, $\mathbf{k}_{e l}^{0}=\left[k_{t}^{0}, k_{n}^{0}\right\rfloor$ is a diagonal stiffness matrix, with $k_{t}^{0}, k_{n}^{0}$ tangential and normal interface elastic moduli, $\psi_{\text {in }}(\eta)$ is the internal energy density governing the softening behaviour in the damage evolution process and it is function of the internal variable $\eta$.

The traction separation law in the undeformed reference can be defined by the following state equation

$$
\tilde{\boldsymbol{T}}=\frac{\partial \psi}{\partial \llbracket \tilde{\boldsymbol{u}} \rrbracket}=(1-\omega) \mathbf{k}_{e l}^{0} \cdot \llbracket \tilde{\boldsymbol{u}} \rrbracket
$$

The elastic behaviour is not assumed isotropic a priori, and $k_{t}^{0}$ and $k_{n}^{0}$ are assumed to be two independent constitutive parameters. The balance of angular momentum can be achieved by assuming the same value for the two parameters $k_{t}^{0}=k_{n}^{0}$ or, as pursued in the present paper, under the hypothesis of small separation displacement before full decohesion.

The traction separation law can also be defined in the current reference, by substitution of Eq.(20) in Eq.(13), that is

$$
\tilde{\boldsymbol{S}}=(1-\omega) \mathbf{k}_{e l} \cdot \llbracket \tilde{\boldsymbol{u}} \rrbracket=(1-\omega) \frac{d \Gamma_{0}}{d \Gamma_{m}} \mathbf{k}_{e l}^{0} \cdot \llbracket \tilde{\boldsymbol{u}} \rrbracket
$$

where $\mathbf{k}_{e l}=\mathbf{k}_{e l}^{0} d \Gamma_{0} / d \Gamma_{m}$ is the matrix of the elastic stiffness parameters in the deformed configuration. Equation (21) shows that the interface elastic parameters cannot be assumed as constant in the deformed configuration but they linearly depend on the ratio between the areas of undeformed surface and deformed surface.

Damage evolution is governed by the following damage activation function

$$
\phi_{d}(Y, \chi)=Y-\chi(\eta)-Y_{0} \leq 0
$$


where the driving activation damage variables respectively are $Y:=-\partial \psi / \partial \omega$ and $\chi:=-\partial \psi / \partial \eta$, being $Y$ the energy release rate given by

$$
Y=\frac{1}{2} \llbracket \tilde{u} \rrbracket \cdot \mathbf{k}_{e l}^{0} \cdot \llbracket \tilde{\boldsymbol{u}} \rrbracket
$$

and $\chi(\eta)$ the static internal variable, which governs the cohesive softening behaviour as function of the kinematic internal variable $\eta$. For linear softening law the internal variable results

$$
\chi(\eta)=\frac{1}{2} k_{n}^{0} u_{e}^{2}\left[\left(\frac{u_{f}}{u_{f}(1-\eta)+u_{e} \eta}\right)^{2}-1\right]
$$

where $u_{e}$ and $u_{f}$ are separation displacement limit values, respectively, at the elastic threshold and at the unitary damage condition, in pure opening condition. The constant term $Y_{0}:=\frac{1}{2} k_{n}^{0} u_{e}^{2}$ is the energy threshold for the initial damage activation. Evolution of damage and internal kinematic variable $\eta$ is governed by the following flow rules and loading-unloading conditions

$$
\begin{aligned}
\dot{\omega} & =\frac{\partial \phi_{d}}{\partial Y} \dot{\lambda}_{d}=\dot{\lambda}_{d} \\
\dot{\eta} & =-\frac{\partial \phi_{d}}{\partial \chi} \dot{\lambda}_{d}=\dot{\lambda}_{d} \\
\dot{\lambda}_{d} & \geq 0, \quad \phi_{d} \dot{\lambda}_{d}=0, \quad \dot{\phi}_{d} \dot{\lambda}_{d}=0
\end{aligned}
$$

with $\dot{\lambda}_{d}$ a damage multiplier. Fracture energy produced by complete delamination is independent of the delamination mode and is defined by $G_{I}=G_{I I}=$ $\frac{1}{2} k_{n}^{0} u_{e} u_{f}$. The evolution of such constitutive model with different mode I and mode II fracture energies has been proposed by the same authors in [21].

\section{Interface finite element formulation}

The interface finite element is developed in the classical isoparametric formulation, depicted in Fig. 3 for a two-dimensional six nodes element with local coordinate $\xi$.

The element domain $\Gamma_{0}^{e}$ is defined by isoparametric mapping

$$
\boldsymbol{X}(\xi)=\frac{1}{2} \sum_{I \in N} \phi_{I}(\xi) \boldsymbol{X}_{I} \text { with } \boldsymbol{X} \in \Gamma_{0}^{e}, \xi \in(-1,1)
$$

where $N$ is the set of element nodes $N=\{1,2,3,4,5,6\}$, as represented in Fig. 3 . We can also define the set of lower side nodes as $N^{-}=\{1,2,3\}$ and the set of upper side nodes as $N^{+}=\{4,5,6\}$. The functions $\phi_{I}(\xi)$ with $I \in N$ are the nodal shape functions and $\boldsymbol{X}_{I}$ with $I \in N$ are the nodal coordinate vectors. Moreover, nodes geometrically coincident in the reference configuration have the same shape function $\left(\phi_{I}(\xi)=\phi_{I+3}(\xi)\right.$ with $I=1,2,3$ in Fig. 3). The current configuration is identified by position of positive and negative sides of 
the interface, and it can can be computed by the same isoparametric mapping by the following relations

$$
\begin{aligned}
& \boldsymbol{x}^{+}\left(\xi, \boldsymbol{u}_{I}\right)=\sum_{I \in N^{+}} \phi_{I}(\xi)\left(\boldsymbol{X}_{I}+\boldsymbol{u}_{I}\right) \\
& \boldsymbol{x}^{-}\left(\xi, \boldsymbol{u}_{I}\right)=\sum_{I \in N^{-}} \phi_{I}(\xi)\left(\boldsymbol{X}_{I}+\boldsymbol{u}_{I}\right)
\end{aligned}
$$

where $\boldsymbol{u}_{I}$ is the nodal displacement vector.

Displacement of positive and negative sides of the interface can be written by the following compact notation

$$
\boldsymbol{u}^{ \pm}\left(\xi, \boldsymbol{u}_{I}\right)=\sum_{I \in N^{ \pm}} \phi_{I}(\xi) \boldsymbol{u}_{I}
$$

and the separation displacement in Eq. (5), in finite element formulation, is given as

$$
\llbracket \boldsymbol{u}\left(\xi, \boldsymbol{u}_{I}\right) \rrbracket=\llbracket \boldsymbol{u} \rrbracket=\sum_{I \in N^{ \pm}} \pm \phi_{I}(\xi) \boldsymbol{u}_{I}
$$

The point at the middle surface in the current configuration and its displacement are defined as

$$
\begin{aligned}
& \boldsymbol{x}_{m}\left(\xi, \boldsymbol{u}_{I}\right)=\frac{1}{2} \sum_{I \in N} \phi_{I}(\xi)\left(\boldsymbol{X}_{I}+\boldsymbol{u}_{I}\right) \\
& \boldsymbol{u}_{m}\left(\xi, \boldsymbol{u}_{I}\right)=\frac{1}{2} \sum_{I \in N} \phi_{I}(\xi) \boldsymbol{u}_{I}
\end{aligned}
$$

The increment vector $d \boldsymbol{x}_{m}$ of the middle surface mapping in the current configuration is related to the increment $d \xi$ by the relation

$$
d \boldsymbol{x}_{m}(\xi)=\frac{1}{2} \sum_{I \in N} \phi_{I, \xi}(\xi)\left(\boldsymbol{X}_{I}+\boldsymbol{u}_{I}\right) d \xi
$$

where $\phi_{I, \xi}(\xi)=d \phi_{I}(\xi) / d \xi$ is the derivative of the I-th shape function with respect to $\xi$. Increment vector $d \boldsymbol{x}_{m}$ is represented in the Figures 3 and 4 , for a two-dimensional problem, as an infinitesimal surface element of area $d \Gamma_{m}$ in the current configuration and of area $d \Gamma_{0}$ in the reference one. Surface element area $d \Gamma_{m}$ is given as

$$
d \Gamma_{m}=\left(d \boldsymbol{x}_{m} \cdot d \boldsymbol{x}_{m}\right)^{\frac{1}{2}}=J_{m}(\xi) d \xi
$$

where $J_{m}(\xi)$ is the norm of the jacobian of isoparametric mapping of the middle surface, defined as

$$
J_{m}(\xi)=\frac{1}{2}\left\|\sum_{I \in N} \phi_{I, \xi}(\xi)\left(\boldsymbol{X}_{I}+\boldsymbol{u}_{I}\right)\right\| .
$$


With reference to Fig. 4, the tangent unit vector $\boldsymbol{t}(\xi)$ can be defined by the following relation

$$
\boldsymbol{t}(\xi)=\frac{d \boldsymbol{x}_{m}(\xi)}{\left\|d \boldsymbol{x}_{m}(\xi)\right\|}=\frac{d \boldsymbol{x}_{m}(\xi)}{d \Gamma_{m}}=\frac{1}{2} \frac{1}{J_{m}} \sum_{I \in N} \phi_{I, \xi}(\xi)\left(\boldsymbol{X}_{I}+\boldsymbol{u}_{I}\right) .
$$

Details of the formulation of geometric operators $J_{m}, \boldsymbol{t}$ and $\boldsymbol{n}$ are analysed

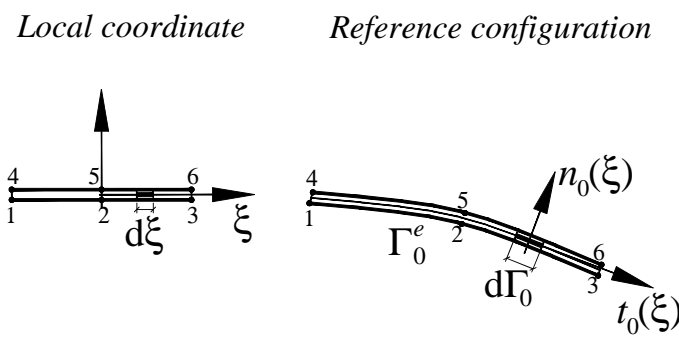

\section{Current configuration}

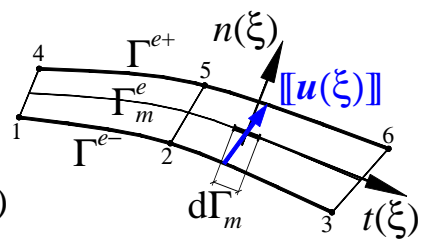

Fig. 3 Representation of a six node isoparametric interface element in the natural reference, in the initial configuration and in the current configuration.

in the Appendix.

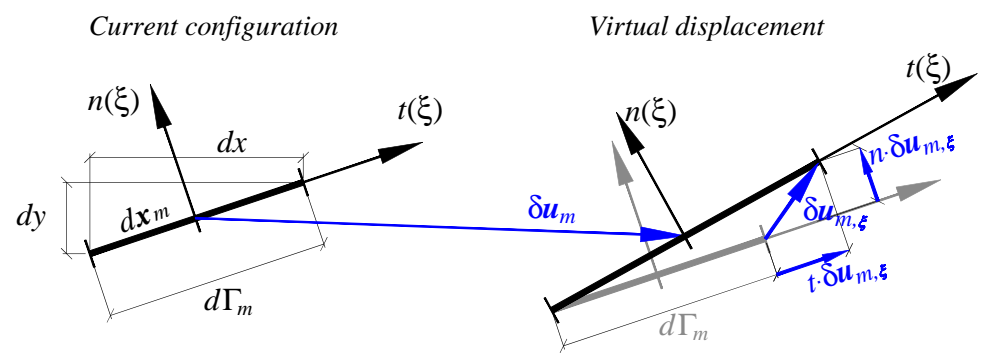

Fig. 4 Representation of the infinitesimal surface element $d \Gamma_{m}$ of the middle surface in the current configuration and its variation produced by a virtual displacement.

4.1 Differentials and derivatives with respect to nodal displacement

The finite element formulation requires the evaluation of derivatives with respect to vector displacement $\boldsymbol{u}_{I}$ or, equivalently, the evaluation of the differentials of all the geometric operators, that are the jacobian norm of middle 
surface mapping, tangent and normal vectors. Considering Eq. (33) and Eq. (34), the variation of norm of middle surface jacobian, due to the virtual nodal displacement $\delta \boldsymbol{u}_{I}$ with $I \in N$, can be defined by the following relation

$$
\delta J_{m}(\xi)=\sum_{I \in N} \frac{\partial J_{m}}{\partial \boldsymbol{u}_{I}} \cdot \delta \boldsymbol{u}_{I}=\boldsymbol{t} \cdot \delta \boldsymbol{u}_{m, \xi}
$$

with

$$
\frac{\partial J_{m}}{\partial \boldsymbol{u}_{I}}=\frac{1}{2} \boldsymbol{t} \phi_{I, \xi} \quad \text { and } \quad \delta \boldsymbol{u}_{m, \xi}=\frac{1}{2} \sum_{I \in N} \phi_{I, \xi} \delta \boldsymbol{u}_{I} .
$$

The effects of a virtual displacement on the surface element $d \Gamma_{m}$ is shown in Fig. 4, where the tangential component of $\delta \boldsymbol{u}_{m, \xi}$ produces an elongation of the surface element $d \Gamma_{m}$ and the normal one produces a rotation of the surface element. Variations of tangential and normal unit vectors, due to a virtual displacement, can be obtained by differentiation of Eq. (34), as following

$$
\begin{aligned}
\delta \boldsymbol{t}(\xi) & =\sum_{I \in N} \frac{\partial \boldsymbol{t}}{\partial \boldsymbol{u}_{I}} \cdot \delta \boldsymbol{u}_{I}=\frac{1}{J_{m}} \boldsymbol{n}\left(\boldsymbol{n} \cdot \delta \boldsymbol{u}_{m, \xi}\right) \\
\delta \boldsymbol{n}(\xi) & =\sum_{I \in N} \frac{\partial \boldsymbol{n}}{\partial \boldsymbol{u}_{I}} \cdot \delta \boldsymbol{u}_{I}=-\frac{1}{J_{m}} \boldsymbol{t}\left(\boldsymbol{n} \cdot \delta \boldsymbol{u}_{m, \xi}\right),
\end{aligned}
$$

with

$$
\begin{gathered}
\frac{\partial \boldsymbol{t}(\xi)}{\partial \boldsymbol{u}_{I}}=\frac{\phi_{I, \xi}}{2 J_{m}} \boldsymbol{n} \otimes \boldsymbol{n} \\
\frac{\partial \boldsymbol{n}(\xi)}{\partial \boldsymbol{u}_{I}}=-\frac{\phi_{I, \xi}}{2 J_{m}} \boldsymbol{t} \otimes \boldsymbol{n} .
\end{gathered}
$$

By considering that $1 / J_{m} \boldsymbol{n} \cdot \delta \boldsymbol{u}_{m, \xi}$ is a scalar value, the equations $(37 \mathrm{a}, \mathrm{b})$ show that the variations of the local axes, due to a virtual nodal displacement, are two $\pi / 2$ rotated (anticlockwise) vectors. Consequently, the variation of rotation matrix $\mathbf{G}$ is defined as

$$
\delta \mathbf{G}(\xi)=\sum_{I \in N} \frac{\partial \mathbf{G}}{\partial \boldsymbol{u}_{I}} \cdot \delta \boldsymbol{u}_{I}=\frac{1}{J_{m}} \mathbf{G}_{r} \sum_{I \in N} \Phi_{I, \xi} \boldsymbol{n} \cdot \delta \boldsymbol{u}_{I}
$$

where $\mathbf{G}_{r}=[\boldsymbol{n},-\boldsymbol{t}]^{T}$ is a new rotational matrix.

Details of derivative of the geometric operators $J_{m}, \boldsymbol{t}$ and $\boldsymbol{n}$ are analysed in the Appendix.

4.2 Weak form equilibrium condition (PVW)

The weak form equilibrium condition is mathematically defined in Eq. (17) in the reference configuration and in Eq. (18) in the current configuration. The contribution of a single interface finite element can be written as

$$
\int_{\Gamma_{0}^{e}} \tilde{\boldsymbol{T}} \cdot \llbracket \tilde{\delta \boldsymbol{u} \rrbracket d \Gamma_{0}}=\int_{\Gamma_{m}^{e}} \tilde{\boldsymbol{S}} \cdot \llbracket \tilde{\delta \boldsymbol{u} \rrbracket} d \Gamma_{m}=\sum_{I \in N} \boldsymbol{q}_{I} \cdot \delta \boldsymbol{u}_{I}
$$


where the nodal force vector $\boldsymbol{q}_{I}$ can be computed in the reference configuration by substitution of Eq. (39) and Eq. (29) in Eq. (17), that is

$$
\boldsymbol{q}_{I}=\int_{-1}^{1} \tilde{\boldsymbol{T}} \cdot\left[\frac{\phi_{I, \xi}}{2 J_{m}}\left(\mathbf{G}_{r} \cdot \llbracket \boldsymbol{u} \rrbracket\right) \otimes \boldsymbol{n} \pm \phi_{I} \mathbf{G}\right] J_{0} d \xi \text { with } I \in N^{ \pm}
$$

where $J_{0}=\frac{1}{2}\left\|\sum_{I \in N} \phi_{I, \xi}(\xi) \boldsymbol{X}_{I}\right\|$ is the jacobian norm of the isoparametric mapping in the reference configuration. Substitution of traction separation law of Eq.(20) in Eq. (41) gives the nodal force vector as function of displacement as

$$
\boldsymbol{q}_{I}=\int_{-1}^{1}(1-\omega) \llbracket \tilde{\boldsymbol{u}} \rrbracket \cdot \mathbf{k}_{e l}^{0} \cdot\left[\frac{\phi_{I, \xi}}{2 J_{m}}\left(\mathbf{G}_{r} \cdot \llbracket \boldsymbol{u} \rrbracket\right) \otimes \boldsymbol{n} \pm \phi_{I} \mathbf{G}\right] J_{0} d \xi \text { with } I \in N^{ \pm}
$$

The nodal force vector $\boldsymbol{q}_{I}$ can be also computed in the deformed configuration by substitution of Eq. (39) and Eq. (29) in Eq. (18), that is

$$
\boldsymbol{q}_{I}=\int_{-1}^{1} \tilde{\boldsymbol{S}} \cdot\left[\frac{\phi_{I, \xi}}{2 J_{m}}\left(\mathbf{G}_{r} \cdot \llbracket \boldsymbol{u} \rrbracket\right) \otimes \boldsymbol{n} \pm \phi_{I} \mathbf{G}\right] J_{m} d \xi \text { with } I \in N^{ \pm} .
$$

In finite element formulation area of infinitesimal surface element is defined in Eq.(33) and the traction separation law in the deformed configuration, defined in Eq. (21), can be written as

$$
\tilde{\boldsymbol{S}}=(1-\omega) \mathbf{k}_{e l} \cdot \llbracket \tilde{\boldsymbol{u}} \rrbracket=(1-\omega) \frac{J_{m}}{J_{0}} \mathbf{k}_{e l}^{0} \cdot \llbracket \tilde{\boldsymbol{u}} \rrbracket,
$$

showing that the nodal forces vector defined in the deformed configuration in Eq.(43) coincides to the nodal forces vector integrated in the reference configuration in Eq.(42).

The Equations (43) and (44) show that the cohesive law can be defined in the current configuration in terms of Cauchy traction vector, which is defined as function of normal and tangential components of separation displacement, and as function of the interface elongation. On the contrary, the use of the same cohesive law in order to define the Piola-Kirchhoff traction vector in the reference configuration and the Cauchy traction vector in the deformed configuration, would produces different numerical solutions between the two integration schemes. The same results has been pointed out in [17], where the integration of the nodal force vector over the reference and over the current configurations, by the use of the same cohesive law, produced different numerical solutions both for elastic interface problems and for delamination interface problems.

\subsection{Consistent stiffness matrix}

Consistent tangent stiffness matrix, required in order to archive a fast asymptotic second order rate of convergence, is defined through derivative of nodal 
force vector in Eq. (41) with respect to nodal displacement, that is

$$
\boldsymbol{K}_{I J}=\frac{\partial \boldsymbol{q}_{I}}{\partial \boldsymbol{u}_{J}}=\boldsymbol{K}_{I J}^{m a t}+\boldsymbol{K}_{I J}^{g e o} \text { with } I, J \in N
$$

where $\boldsymbol{K}_{I J}^{m a t}$ is the material stiffness matrix and $\boldsymbol{K}_{I J}^{g e o}$ is the geometric stiffness matrix.

The material stiffness matrix is defined as

$$
\boldsymbol{K}_{I J}^{m a t}=\int_{-1}^{1} \frac{\partial \tilde{\boldsymbol{T}}}{\partial \boldsymbol{u}_{J}} \cdot\left[\frac{\phi_{I, \xi}}{2 J_{m}}\left(\mathbf{G}_{r} \cdot \llbracket \boldsymbol{u} \rrbracket\right) \otimes \boldsymbol{n} \pm \phi_{I} \mathbf{G}\right] J_{0} d \xi
$$

where the cohesive law is defined in the local reference as $\tilde{\boldsymbol{T}}(\llbracket \tilde{\boldsymbol{u}} \rrbracket)$ and, by considering Eq. (15), its derivative can be defined as

$$
\frac{\partial \tilde{\boldsymbol{T}}}{\partial \boldsymbol{u}_{J}}=\frac{\partial \tilde{\boldsymbol{T}}}{\partial \llbracket \tilde{\boldsymbol{u}} \rrbracket} \cdot \frac{\partial \llbracket \tilde{\boldsymbol{u}} \rrbracket}{\partial \boldsymbol{u}_{J}}=\tilde{\boldsymbol{k}}^{c n} \cdot\left(\frac{1}{J_{m}}\left(\mathbf{G}_{r} \cdot \llbracket \boldsymbol{u} \rrbracket\right) \otimes \boldsymbol{n} \frac{1}{2} \phi_{J, \xi} \pm \mathbf{G} \phi_{J}\right)
$$

where $\tilde{\boldsymbol{k}}^{c n}=\partial \tilde{\boldsymbol{T}} / \partial \llbracket \tilde{\boldsymbol{u}} \rrbracket$ is the interface tangent constitutive matrix. Substitution of Eq. (47) in Eq. (46) allows to write the material stiffness matrix, that is

$$
\begin{aligned}
\boldsymbol{K}_{I J}^{\text {mat }}= & \int_{-1}^{1}\left(\frac{1}{J_{m}} \boldsymbol{n} \otimes\left(\llbracket \boldsymbol{u} \rrbracket \cdot \mathbf{G}_{r}^{T}\right) \frac{1}{2} \phi_{I, \xi} \pm \mathbf{G}^{T} \phi_{I}\right) \cdot \tilde{\boldsymbol{k}}^{c n} . \\
& \cdot\left(\frac{1}{J_{m}}\left(\mathbf{G}_{r} \cdot \llbracket \boldsymbol{u} \rrbracket\right) \otimes \boldsymbol{n} \frac{1}{2} \phi_{j, \xi} \pm \mathbf{G} \phi_{J}\right) J_{0} d \xi \text { with } I, J \in N^{ \pm},
\end{aligned}
$$

whose symmetry condition depends on the tangent constitutive matrix $\tilde{\boldsymbol{k}}^{c n}$.

The term in square parenthesis in Eq. (41) is the sum of rotational matrix $\mathbf{G}$ and of derivative of $\mathbf{G}$ with respect to $\boldsymbol{u}$ (see Eq. (16)) and its derivative involves second order derivative of rotational matrix. In the present paper the second order derivative of rotational matrix $\mathbf{G}$ is assumed negligible and omitted and the geometric stiffness matrix is defined as

$$
\boldsymbol{K}_{I J}^{g e o}=\int_{-1}^{1} \tilde{\boldsymbol{T}} \cdot\left[\mathbf{G}_{r} \otimes \boldsymbol{n} \frac{1}{J_{m}}\left( \pm \phi_{I}\right) \frac{1}{2} \phi_{J, \xi}\right] J_{0} d \xi \text { with } I, J \in N^{ \pm},
$$

which is not a symmetric matrix.

\section{Numerical simulation}

The finite displacement interface formulation developed in the present paper and the assumed interface constitutive model have been implemented in the open source finite element code FEAP [19] for a two dimensional six-nodes interface element and the numerical simulation of the the end-notched double cantilever beam test (DCBT) has been performed.

Sizes and geometry of analysed specimen are depicted in Fig. 5. The lower arm is constrained and the upper one is subjected to imposed displacement $u$ 
in a fixed direction. For a vertical imposed displacement $(\gamma=0$ in Fig. 5) the classical DCB test is performed, whereas a non-vertical displacement produces a rigid finite rotation, over the classical crack opening. However, the mechanical responses, in terms of applied load $P$ and crack opening displacement $d$ is independent on any rigid rotation and, as a consequence, has to be independent on the direction of the imposed displacement $u$.

The modified DCBT, with non-vertical displacement, is proposed in the present paper in order to have a significant modified current configuration, with respect to the reference one. The mechanical response of the modified DCBT can be correctly performed only under finite displacement formulation.

The analytical response, with vertical displacement, is known under small displacement bending beam theory and linear fracture mechanics theory (see [22]), and it is given, in terms of crack opening displacement $d$ and relevant load $P$, as

$$
\begin{aligned}
d & =4 a^{2} \sqrt{\frac{G_{I}}{3 E h^{3}}} \\
P & =\frac{3 E I}{2 a^{3}} d
\end{aligned}
$$

with: $I=b h^{3} / 12, a$ the crack length, $E$ Young modulus and $G_{I}$ fracture energy.

The numerical simulations have been performed using $2 \mathrm{D}$ nine nodes elements and six nodes interface elements. The bulk is modeled by finite deformation neo-Hookean hyperelastic model with Young modulus $E=35300 \mathrm{~N} / \mathrm{mm}^{2}$ and Poisson ratio $\nu=0.27$ (standard parameters for E-glass/epoxy composite material).

The interface model is defined by the following constitutive parameters: $k_{n}=k_{t}=50 \mathrm{KN} / \mathrm{mm}^{3}, u_{e}=2 \cdot 10^{-4} \mathrm{~mm}$ and $u_{f}=0.2 \mathrm{~mm}$. The fracture energy is $G_{I}=\frac{1}{2} k_{n} u_{e} u_{f}=1 \mathrm{~N} / \mathrm{mm}$.

Results of numerical simulation are plotted in Fig. 6 in terms of maximum principal Cauchy stress, in the current configuration, for an horizontal imposed displacement ( $\gamma=\pi / 2$ in Fig. 5), at fives different loading steps: a) $u_{x}=5 \mathrm{~mm}$; b) $u_{x}=10 \mathrm{~mm}$; c) $u_{x}=15 \mathrm{~mm}$; d) $u_{x}=20 \mathrm{~mm}$; e) $u_{x}=25 \mathrm{~mm}$. Results of numerical simulations with three different loading angles $\gamma$ are compared in Figure 7 with results of small displacement solution and analytical solution, in terms of applied load $P$ vs crack opening displacement $d$. The good agreement between the different solutions can be appreciated, excluding the initial elastic branch for well known motivations induced by elastic beam theory [21].

The developed high order interface finite element is considered for a large displacement convergence test by analysing four different meshes of the specimen subjected to a horizontal imposed displacement. The mesh a) is composed of 480 nine-node quadrilateral elements, 76 six-node interface elements and 2254 nodes; the mesh b) is composed of 1600 nine-node quadrilateral elements, 150 six-node interface elements and 7062 nodes; the mesh c) is composed of 2400 nine-node quadrilateral elements, 230 six-node interface elements and 


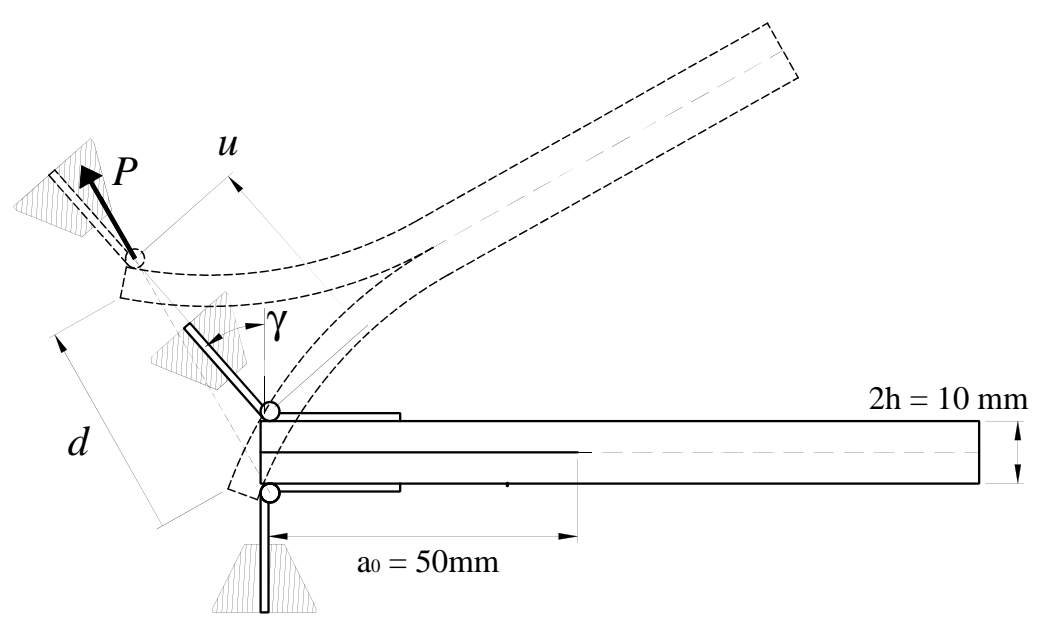

Fig. 5 Sizes and geometry of specimen for the modified double cantilever beam test, with a horizontal imposed displacement.

10582 nodes; the mesh d) is composed of 960 six-node triangular elements, 76 six-node interface elements and 2254 nodes. The results of the four numerical simulations are compared in Fig. 8 with the analytical solution in terms of applied load $P$ vs crack opening displacement $d$. Figure 8 shows that the numerical results obtained with the four different meshs are almost coincident. Results of numerical simulations are plotted in Fig. 9 in terms of maximum principal Cauchy stress at the cohesive process zone, in the current configuration, for the four different meshes.

\section{Closing remarks}

The paper propose a rigorous formulation of interface finite element, developed under finite displacements hypothesis and assuming, as local reference frame of the constitutive model, normal and tangential axes to the middle surface between positive and negative edges of the interface in the deformed configuration. The paper discusses the problem of the correct integration of nodal forces vector and stiffness matrix over the reference configuration and over the deformed one. The relation between the cohesive laws in the reference configuration and in the current configuration is analysed. The constitutive model is based on the an Helmoltz free energy density function, per unit of undeformed interface surface, which allows to define the cohesive law in the reference configuration in terms the Piola-Kirchhoff traction vector, as function of separation displacement and some internal variables. 
a)

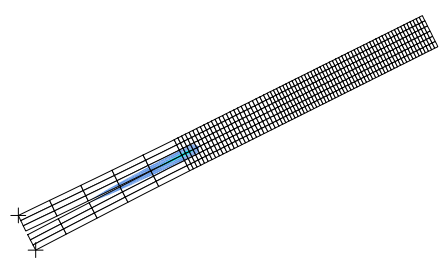

b)

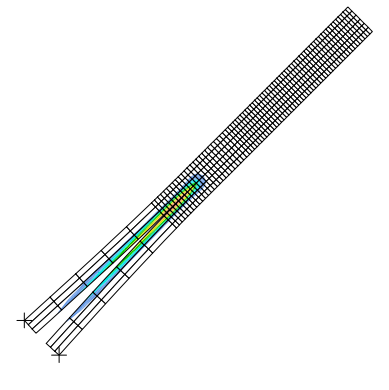

PRIN. STRESS $1[\mathrm{~N} / \mathrm{mm} 2]$

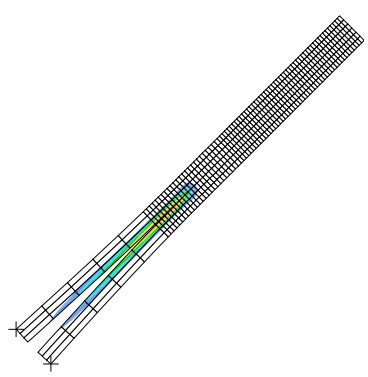

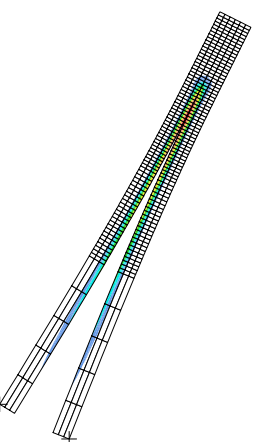

d)

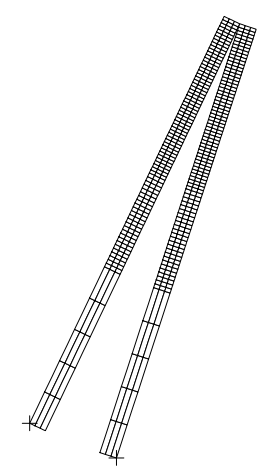

e)

Fig. 6 Map of maximum principal stress in the current configuration, obtained by the numerical simulation of the double cantilever beam test, for an horizontal displacement, at fives different loading steps: a) $u_{x}=5 \mathrm{~mm}$; b) $u_{x}=10 \mathrm{~mm}$; c) $u_{x}=15 \mathrm{~mm}$; d) $u_{x}=20 \mathrm{~mm}$; e) $u_{x}=25 \mathrm{~mm}$.

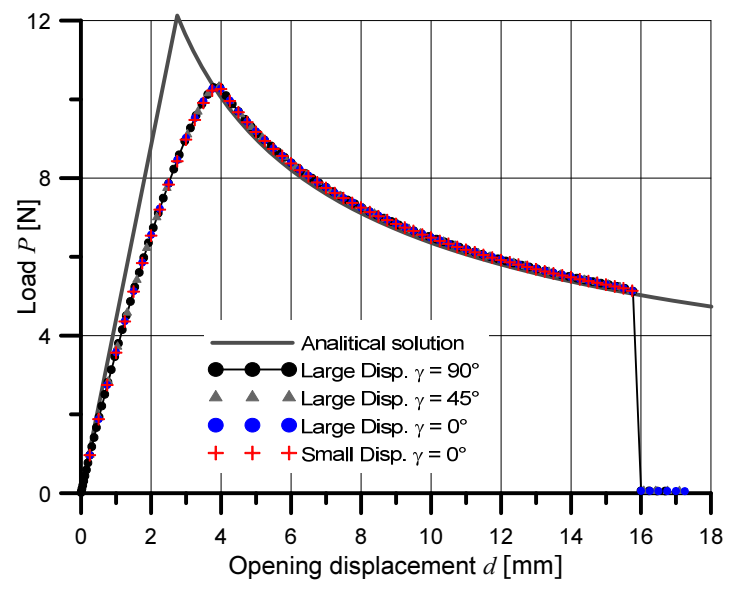

Fig. 7 Response of double cantilever beam test, in terms of applied load vs crack opening displacement. Analytical solution, large displacement numerical solutions for three different displacement directions and small displacement numerical solution with vertical displacement. 


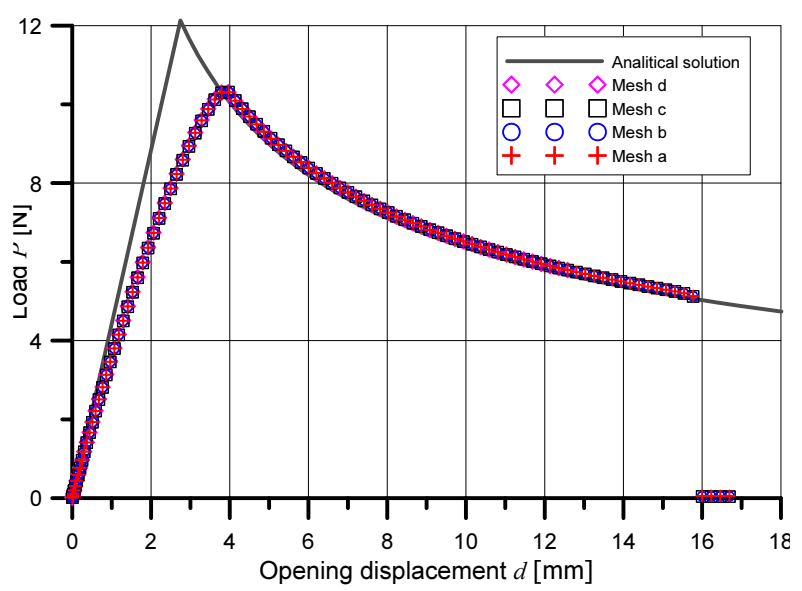

Fig. 8 Convergence test for the double cantilever beam test. Results of the numerical simulations for the four analysed meshes to the horizontal imposed displacement. The results are plotted in terms of applied load vs crack opening displacement and are compared to the analytical solution.
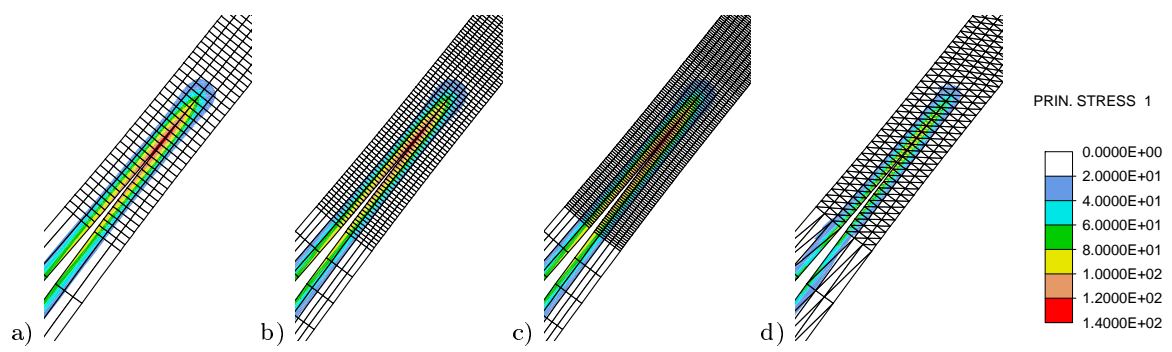

Fig. 9 Map of maximum principal stress in the current configuration at the cohesive process zone, obtained for four meshes by the numerical simulation of the double cantilever beam test, for an horizontal displacement.

It has been shown that the cohesive law can be defined in the current configuration in terms of Cauchy traction vector, which is defined as function of normal and tangential components of separation displacement, and also depends on the interface elongation. On the contrary, the use of the unmodified cohesive law in order to define the Piola-Kirchhoff traction vector in the reference configuration, and the Cauchy traction vector in the deformed configuration, would produces different numerical results between the two integration schemes. In [17] the inconsistency of the numerical integration has been raised, but a specific analysis or explanation on the very nature of the problem has not been provided; neither the related corrections have been pointed out.

The results of an end-notched double cantilever beam test with four different meshes are proposed showing a good agreement with the analytical solution. 


\section{A Derivative of geometric operators}

In this appendix, the partial derivatives of geometric operators $J_{m}, \boldsymbol{t}$ and $\boldsymbol{n}$, with respect to nodal displacements, are analysed by use of index notation. The geometric operators are referred to the Cartesian coordinate axes $X_{i}$, with $(i=1,2)$ for a two-dimensional problem. The norm of middle surface mapping in Eq.(33), with summation of repeated index $i$, can be rewritten as

$$
J_{m}(\xi)=\frac{1}{2}\left[\sum_{I \in N} \phi_{I, \xi}\left(X_{I i}+u_{I i}\right) \sum_{J \in N} \phi_{J, \xi}\left(X_{J i}+u_{J i}\right)\right]^{\frac{1}{2}},
$$

where $X_{I i}$ and $u_{I i}$ are, respectively, the i-th coordinate in the reference configuration and the i-th displacement component of node $I \in N$. The partial derivative of $J_{m}$ is

$$
\frac{\partial J_{m}}{\partial u_{I i}}=\frac{1}{4} \frac{1}{J_{m}} \sum_{J \in N} \phi_{J, \xi}\left(X_{J i}+u_{J i}\right) \phi_{I, \xi}
$$

and by considering the tangent unit vector in Eq. (34) in the following index notation

$$
t_{i}=\frac{1}{2} \frac{1}{J_{m}} \sum_{I \in N} \phi_{I, \xi}\left(X_{I i}+u_{I i}\right)
$$

Eq. (52) can be written as

$$
\frac{\partial J_{m}}{\partial u_{I i}}=\frac{1}{2} t_{i} \phi_{I, \xi},
$$

which represent the index notation of Eq. (25a).

The partial derivative of the tangent unit vector $t$ in Eq. (53), with respect to the nodal displacement component, is

$$
\frac{\partial t_{i}}{\partial u_{I j}}=\frac{1}{2} \frac{\phi_{I, \xi}}{J_{m}} \delta_{i j}-\frac{1}{2} \frac{1}{J_{m}^{2}} \sum_{J \in N} \phi_{J, \xi}\left(X_{J i}+u_{J i}\right) \frac{\partial J_{m}}{\partial u_{I j}}
$$

where $\delta_{i j}$ is the Kronecker delta. Substitution of Eqs. (53) and (54) in Eq. (55) gives the following equation

$$
\frac{\partial t_{i}}{\partial u_{I j}}=\frac{1}{2} \frac{\phi_{I, \xi}}{J_{m}}\left(\delta_{i j}-t_{i} t_{j}\right)=\frac{1}{2} \frac{\phi_{I, \xi}}{J_{m}} n_{i} n_{j},
$$

The latter equality in Eq. (56) is based on the relations between normal and tangent unit vectors $\left(n_{1}=t_{2}\right.$ and $\left.n_{2}=-t_{1}\right)$ and it is equivalent to Eq. (38a).

Derivative of normal unit vector can be obtained by the relations between normal and tangent unit vectors and it gives

$$
\frac{\partial n_{i}}{\partial u_{I j}}=-\frac{1}{2} \frac{\phi_{I, \xi}}{J_{m}} t_{i} n_{j}
$$

which is equivalent to Eq. (38b).

\section{Compliance with Ethical Standards}

Funding: The financal support of the Italian Ministry for University and Research (MIUR), under the grant PRIN-2015, project No.2015LYYXA8, "Multiscale mechanical models for the design and optimization of microstructured smart materials and metamaterials" is gratefully acknowledged. 


\section{Conflict of Interest}

The authors declare that they have no conflict of interest.

\section{References}

1. O. Allix, A. Corigliano, Geometrical and interfacial non-linearities in the analysis of delamination in composites, International Journal of Solids and Structures 36 (15) (1999) $2189-2216$.

2. G. Scimemi, G. Giambanco, A. Spada, The interphase model applied to the analysis of masonry structures, Computer Methods in Applied Mechanics and Engineering 279 (2014) 66-85. doi:10.1016/j.cma.2014.06.026.

3. E. Sacco, J. Toti, Interface elements for the analysis of masonry structures, International Journal of Computational Methods in Engineering Science and Mechanics 11 (6) (2010) 354-373. doi:10.1080/15502287.2010.516793.

4. G. Borino, L. Fratini, F. Parrinello, Mode i failure modeling of friction stir welding joints, International Journal of Advanced Manufacturing Technology 41 (5-6) (2009) 498-503. doi:10.1007/s00170-008-1498-1

5. G. Camacho, M. Ortiz, Computational modelling of impact damage in brittle materials, International Journal of Solids and Structures 33 (20) (1996) 2899 - 2938. doi:http://dx.doi.org/10.1016/0020-7683(95)00255-3.

6. A. Pandolfi, P. Krysl, M. Ortiz, Finite element simulation of ring expansion and fragmentation: The capturing of length and time scales through cohesive models of fracture, International Journal of Fracture 95 (1) (1999) 279-297. doi:10.1023/A:1018672922734.

7. J. Mosler, I. Scheider, A thermodynamically and variationally consistent class of damage-type cohesive models, J. Mech. Physics Solids 59 (8) (2011) 1647-1668.

8. N. Ottosen, M. Ristinmaa, J. Mosler, Fundamental physical principles and cohesive zone models at finite displacements - limitations and possibilities, International Journal of Solids and Structures 53 (2015) 70-79, cited By 0. doi:10.1016/j.ijsolstr.2014.10.020.

9. B. Vossen, P. Schreurs, O. van der Sluis, M. Geers, On the lack of rotational equilibrium in cohesive zone elements, Computer Methods in Applied Mechanics and Engineering 254 (2013) $146-153$.

10. M. Ortiz, A. Pandolfi, Finite-deformation irreversible cohesive elements for threedimensional crack-propagation analysis, International Journal for Numerical Methods in Engineering 44 (9) (1999) 1267-1282.

11. Y. Qiu, M. Crisfield, G. Alfano, An interface element formulation for the simulation of delamination with buckling, Engineering Fracture Mechanics 68 (16) (2001) 1755-1776. doi:10.1016/S0013-7944(01)00052-2.

12. M. van den Bosch, P. Schreurs, M. Geers, A cohesive zone model with a large displacement formulation accounting for interfacial fibrilation, European Journal of Mechanics - A/Solids 26 (1) (2007) 1 - 19.

13. V. Tvergaard, Effect of fibre debonding in a whisker-reinforced metal, Materials Science and Engineering: A 125 (2) (1990) $203-213$.

14. P. H. Geubelle, J. S. Baylor, Impact-induced delamination of composites: a 2d simulation, Composites Part B: Engineering 29 (5) (1998) 589 - 602.

15. M. van den Bosch, P. Schreurs, M. Geers, An improved description of the exponential $\mathrm{xu}$ and needleman cohesive zone law for mixed-mode decohesion, Eng. Fract. Mech. 73 (9) (2006) 1220-1234.

16. J. Reinoso, M. Paggi, A consistent interface element formulation for geometrical and material nonlinearities, Computational Mechanics 54 (6) (2014) 1569-1581. doi:10.1007/s00466-014-1077-2.

17. P. Gilormini, J. Diani, Testing some implementations of a cohesive-zone model at finite strain, Engineering Fracture Mechanics 148 (2015) 97 - 109 doi:http://dx.doi.org/10.1016/j.engfracmech.2015.09.013.

18. K. Park, G. Paulino, J. Roesler, A unified potential-based cohesive model of mixed-mode fracture, J. Mech. Physics Solids 57 (6) (2009) 891-908. 
19. O. Zienkiewicz, Taylor, The Finite Element Method. 5th Edition, ButterworthHeinemann Press, 2000.

20. F. Parrinello, B. Failla, G. Borino, Cohesive-frictional interface constitutive model, Int. J. Solids Structures 46 (13) (2009) 2680-2692.

21. F. Parrinello, G. Marannano, G. Borino, A thermodynamically consistent cohesivefrictional interface model for mixed mode delamination, Engineering Fracture Mechanics 153 (2016) 61-79, cited By 0. doi:10.1016/j.engfracmech.2015.12.001.

22. E. Gdoutos, Fracture Mechanics. An introduction, Kluver Academic Publishers, 1993. 